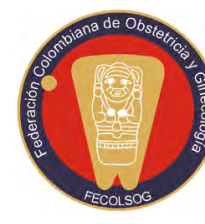

\title{
LESIONES PRECURSORAS DEL CÁNCER ANAL EN LA MUJER: ABORDAJE DESDE LA ÓPTICA DEL GINECÓLOGO
}

a infección de transmisión sexual más frecuente en el mundo es la causada por el virus del papiloma humano (VPH). Para 2010 se estimaba que en mujeres con hallazgos citológicos normales la prevalencia global de infección por VPH estaba entre el 7 y el $11 \%$, mientras en América Latina y el Caribe la prevalencia era del $16 \%$, una de las más altas a nivel mundial (1). El VPH es la segunda causa infecciosa de cáncer después de la bacteria Helicobacter pylori (2). Se estima que para el periodo 2013-2017 se presentaron cerca de 45.300 casos nuevos de cáncer asociados al VPH en Estados Unidos, especialmente cáncer anogenital y orofaríngeo (3).

Los cánceres en donde se ha encontrado un mayor riesgo atribuible al VPH son el de cérvix (100\%) y el anal (88\%) (4). El carcinoma anal de células escamosas es causado especialmente por los serotipos 16 y 18, considerados de alto riesgo oncogénico (4), ya que en biopsias de neoplasias escamosas anales de poblaciones europeas, asiáticas y norteamericanas se ha identificado presencia de ADN de VPH, principalmente de subtipos 16 (80\%) y 18 (10\%) (5). El cáncer anal representa aproximadamente del 1 al $2 \%$ de los cánceres gastrointestinales y se estima que en Estados Unidos cada año aparecen cerca de 4.700 casos nuevos de cáncer anal asociado al VPH en mujeres, y unos 2.300 en hombres (2). En Colombia, se ha descrito que la incidencia ajustada por edad para el periodo 2010-2012 variaba del 0,5 al 1,9 x 100.000 mujeres año y del 0,5 al 0,9 en 100.000 hombres año (6). En cuanto a la mortalidad por cáncer anal, se estima que en Estados Unidos aproximadamente 1.350 personas habrán muerto por este cáncer en 2020 (7).
Se ha informado que las mujeres con historia de neoplasia intraepitelial del cérvix (NIC) tienen mayor riesgo de infección anal por VPH de alto riesgo oncogénico que aquellas sin NIC (17,4 vs. 1,5\% respectivamente) y que este virus infecta la mucosa cervical y anal en el $4 \%$ de las mujeres con NIC (8). Además, hay evidencia de que las mujeres con historia de NIC grado 3 tienen mayor riesgo de desarrollar cáncer anal con una razón de tasa de incidencia (RTI) de 3,85 (IC $95 \%$ : 2,32-6,37) y de desarrollar NIA grado 3 (RTI: 6,68; (IC 95\%: 3,64-12,25) (9)

Entendida en este contexto, la neoplasia intraepitelial anal (NIA) forma parte de una enfermedad multicéntrica en relación con el tracto genital inferior y, por tanto, la presencia de neoplasia intraepitelial del cérvix, vagina y vulva puede ser una señal que alerte sobre la presencia o futura aparición de lesiones intraepiteliales anales (10). En Colombia, pocos estudios han evaluado la prevalencia de la neoplasia intraepitelial anal en mujeres con displasia del tracto genital inferior. En 2018, Gomez et al. publicaron un estudio de 119 mujeres con diagnóstico histopatológico de NIC2/3 y carcinoma in situ, encontrando una prevalencia de NIA de 6,7\% (7 mujeres con NIA de bajo grado y una con NIA de alto grado). Se encontró infección por VPH anal en 45 mujeres (37,8\%), con predominio de los genotipos 16, 18 de VPH (11).

Este panorama de mayor riesgo de cáncer anal en la mujer, sobre todo en aquellas con antecedente de NIC, contrasta con el poco interés que despierta en los ginecólogos esta patología del área perineal y perianal. El presente número de la RCOG presenta el estudio exploratorio de la prevalencia de infección por 
VPH de alto riesgo en la mucosa anal de mujeres con diagnóstico reciente de NIC en la ciudad de Bogotá. La importancia de este estudio radica en llamar la atención a entes gubernamentales, aseguradoras, a médicos especialistas en ginecología y obstetricia y a los investigadores sobre el riesgo de NIA presente en las mujeres con antecedente de displasia del tracto genital inferior de cualquier grado.

Para los primeros, es relevante evaluar si es efectivo y seguro implementar guías para la prevención y el diagnóstico temprano de la infección anal por VPH de alto riesgo y de la NIA en mujeres de alto riesgo para cáncer anal (antecedente de NIC y con infección por VIH) (12). Para los médicos ginecobstetras es relevante advertir a las pacientes sobre esta posibilidad y reconocer la necesidad de ampliar el horizonte del exámen clínico ginecológico anual de manera que la evaluación del área perianal sea rutinaria y se conozca la utilidad de las diferentes alternativas de tamizaje de la NIA. Vale la pena recordar que las pruebas más usadas en la literatura para la tamización y el diagnóstico de NIA son la citología anal, reportada con el sistema Bethesda, junto con el examen digital ano-rectal (en inglés conocido por el acrónimo DARE) (12) y la anoscopia de alta resolución (13). Además, se requiere que en las unidades de patología del tracto genital inferior y colposcopia se considere la conformación de grupos multidisciplinarios para el diagnóstico y la atención de las mujeres con la patología premaligna del tracto genital inferior que incluye a la NIA.

Por último, es necesario que los investigadores en la patología del tracto genital inferior realicen estudios en poblaciones de alto riesgo sobre la frecuencia de la infección anal por VPH 16 y 18, y que también evaluen la exactitud de las pruebas de tamización y su efectividad, seguridad y aceptabilidad en nuestro medio. Del mismo modo, debe evaluarse el papel de la vacunación contra el VPH de alto riesgo para la prevención de esta entidad (14).

Vale la pena recordar que muchas mujeres ponen toda su confianza en el ginecobstetra como médico de atención primaria y esperan de él orientación sobre cómo cuidar su salud y que este realice las intervenciones de prevención o detección temprana de las condiciones mórbidas que las puedan afectar.

\section{Jorge Andrés Rubio-Romero}

Editor Asociado

Revista Colombiana de Obstetricia y

Ginecología (RCOG)

\section{Luz Amparo Díaz-Cruz}

Profesora Asociada

Departamento de Obstetricia y Ginecología

Universidad Nacional de Colombia

\section{REFERENCIAS}

1. Bruni L, Diaz M, Castellsagué X, Ferrer E, Bosch FX, de Sanjosé S. Cervical human papillomavirus prevalence in 5 continents: Meta-analysis of 1 million women with normal cytological findings. J Infect Dis. 2010;202(12):1789-99. https://doi. org/10.1086/657321

2. de Martel C, Georges D, Bray F, Ferlay J, Clifford GM. Global burden of cancer attributable to infections in 2018: A worldwide incidence analysis. Lancet Glob Health. 2020;8(2):e180-e190. https://doi. org/10.1016/S2214-109X(19)30488-7

3. Centers for Disease Control and Prevention. Cancers associated with human papillomavirus, United States-2013-2017. United States Cancer Statistics Data Brief. 2020;18. Disponible en: https://www. cdc.gov/cancer/uscs/pdf/USCS-DataBrief-No18September2020-h.pdf

4. de Martel C, Plummer M, Vignat J, Franceschi S. Worldwide burden of cancer attributable to HPV by site, country and HPV type. Int J Cancer. 2017;141(4):66470. https://doi.org/10.1002/ijc.30716

5. Alemany L, Saunier M, Alvarado-Cabrero I, Quirós B, Salmeron J, Shin HR, et al. Human papillomavirus DNA prevalence and type distribution in anal carcinomas worldwide. Int J Cancer. 2015;136(1):98107. https://doi.org/10.1002/ijc.28963 
6. Bruni L, Albero G, Serrano B, Mena M, Gómez D, Muñoz J, et al. Human papillomavirus and related diseases in the world - Summary report. ICO/IARC Inf Cent HPV Cancer (HPV Inf Centre). 2019;307. Disponible en: https:/hpvcentre.net/statistics/reports/ XWX.pdf

7. National Cancer Institute. Surveillance, epidemiology and end results program. Cancer Stat Facts: Anal Cancer. 2018. Disponible en: https://seer.cancer.gov/ statfacts/html/anus.html

8. Bregar AJ, Cronin B, Luis C, DiSilvestro P, Schechter S, Pisharodi L, et al. Anal and cervical high-risk human papillomavirus genotyping in women with and without genital neoplasia. J Low Genit Tract Dis. 2018;22(2):115-9. https://doi.org/10.1097/ LGT.0000000000000368

9. Ebisch RMF, Rutten DWE, IntHout J, Melchers WJG, Massuger LFAG, Bulten J, et al . Long-lasting increased risk of human papillomavirus-related carcinomas and premalignancies after cervical intraepithelial neoplasia grade 3: A population-based cohort study. J Clin Oncol. 2017;35(22):2542-50. https://doi.org/10.1200/ JCO.2016.71.4543

10. Tatti S, Suzuki V, Fleider L, Maldonado V, Caruso $\mathrm{R}$, Tinnirello $\mathrm{M}$ de L. Anal intraepithelial lesions in women with human papillomavirus-related disease. J
Low Genit Tract Dis. 2012;16(4):454-9. https://doi. org/10.1097/LGT.0b013e31825d2d7a

11. Gómez S, Castillo M, Amaya J, Restrepo O, Puentes E y Sanabria D. Prevalencia de neoplasia intraepitelial, infección por VPH y alteraciones en citología anal en mujeres con LEI de alto grado y cáncer in situ de cérvix en una población de Bogotá. Trabajos ganadores presentados en el XXXI Congreso Nacional de Obstetricia y Ginecología realizado del 9 al 12 de mayo de 2018 en Cartagena, Colombia. Rev Colomb Obstet Ginecol. 2018;69(3):218-21. Disponible en: https:// revista.fecolsog.org/index.php/rcog/article/view/3234

12. Alam NN, White DA, Narang SK, Daniels IR, Smart NJ. Systematic review of guidelines for the assessment and management of high-grade anal intraepithelial neoplasia (AIN II/III). Colorectal Dis. 2016;18(2):135-46. https://doi.org/10.1111/codi.13215

13. Bull-Henry K, Morris B, Buchwald UK. The importance of anal cancer screening and high-resolution anoscopy to gastroenterology practice. Curr Opin Gastroenterol. 2020;36(5):393-401. https://doi.org/10.1097/ MOG.0000000000000661

14. Roden RBS, Stern PL. Opportunities and challenges for human papillomavirus vaccination in cancer. Nat Rev Cancer. 2018;18(4):240-54. https://doi.org/10.1038/ nrc. 2018.13 